\title{
Mutual coupling compensation based on direct data domain algorithms
}

\author{
Ali Azarbar ${ }^{1 a)}$, Gholam R Dadashzadeh ${ }^{2}$, and Hamid R Bakhshi' \\ ${ }^{1}$ Islamic Azad University, Science and Reaserch Branch \\ Hesarak, Poonak, Tehran, Iran \\ ${ }^{2}$ Faculty of Engineering, Shahed University, Tehran, Iran \\ a)aliazarbar@piau.ac.ir
}

\begin{abstract}
In this paper, we present an adaptive algorithm based on direct data domain which can compensate the mutual coupling with only one snapshot of the received signals. Without any auxiliary sensors and calibration sources, this algorithm can accurately estimate the coupling coefficients and recover the desired signal. Numerical simulation shows that the recovery of the desired signal is accurate in the presence of mutual coupling.
\end{abstract}

Keywords: mutual coupling, adaptive algorithm

Classification: Microwave and millimeter wave devices, circuits, and systems

\section{References}

[1] I. J. Gupta and A. A. Ksienski, "Effect of mutual coupling on the performance of adaptive array," IEEE Trans. Antennas Propag., vol. 31, pp. 785-791, 1983.

[2] E. Friel and K. Pasala, "Effects of mutual coupling on performance of STAP antenna array," IEEE Trans. Aerosp. Electron. Syst., vol. 36, pp. 518-527, 2000.

[3] B. Friedlander and A. J. Wiss, "Direction finding in the presence of mutual coupling," IEEE Trans. Antennas Propag., vol. 39, pp. 273-284, 1991.

[4] H. Yamada, M. Morishita, and Y. Yamaguchi, "A Simple Mutual Coupling Compensation Technique in Array of Single-Mode Elements by a Weighted Mutual Coupling Matrix Based on the Impedance Matrix," IEICE Trans. Commun., vol. E90-b, pp. 2288-2296, 2007.

[5] R. S. Adve, "Elimination of the Effects of Mutual Coupling in Adaptive Thin Wire Antennas," Ph.D. dissertation, Syracuse Univ., Syracuse, NY, 1996.

[6] H. Aoyamada and H. Arai, "Mutual Coupling Matrix Estimation and Null Forming Methods for MBF Antennas," IEICE Trans. Commun., vol. E88-b, pp. 2305-2312, 2005.

[7] E. Lau, R. Adve, and T. Sarkar, "Minimum Norm Mutual Coupling compensation with Applications in Direction of Arrival estimation," IEEE Trans. Antennas Propag., vol. 52, no. 8, 2004. 
[8] Z. Huang, C. A. Balanis, and C. R. Britcher, "Mutual Coupling Compensation in UCAs," IEEE Trans. Antennas Propag., vol. 54, no. 11, 2006.

[9] T. T. Zhang, Y. L. Lu, and H. Hui, "Compensation for Mutual Coupling Effect in Uniform Circular Arrays for 2D DOA Estimations Employing the Maximum Likelihood Technique," IEEE Trans. Aerosp. Electron. Syst., vol. 44, no. 3, 2008.

[10] H. S. Lui and H. T. Hui, "Effective Mutual Coupling compensation for direction-of-arrival estimations using a new, accurate determination method for the receiving mutual impedance," J. Electromagn. Waves Appl., vol. 24, pp. 271-281, 2010.

[11] B. Wang, Y. Wang, and Y. Guo, "Mutual coupling calibration with instrumental sensors," Electron. Lett., vol. 40, no. 7, 2004.

[12] Y. Horiki and E. H. Newman, "A Self-Calibration Technique for a DOA Array With Near-Zone Scatterers," IEEE Trans. Antennas Propag., vol. 54, no. 4, 2006.

[13] F. Sellone and A. Serra, "A Novel Online Mutual Coupling Compensation Algorithm for ULAs," IEEE Trans. Signal Process., vol. 55, no. 2, 2007.

[14] Z. Ye and C. Liu, "On the Resiliency of MUSIC Direction Finding Against Antenna Sensor Coupling," IEEE Trans. Antennas Propag., vol. 56, no. 2, 2008.

[15] Z. Ye and C. Liu, "2D DOA Estimation in the Presence of Mutual Coupling," IEEE Trans. Antennas Propag., vol. 56, no. 10, 2008.

[16] T. K. Sarkar and N. Sangruji, "An adaptive nulling system for a narrowband signal with a look direction constraint utilizing the conjugate gradient method," IEEE Trans. Antennas Propag., vol. 37, no. 7, 1989.

[17] T. K. Sarkar, H. Wang, S. Park, J. Koh, R. Adve, K. Kim, Y. Zhang, M. C. Wicks, and R. D. Brown, "A deterministic least-squares approach to space time adaptive processing," IEEE Trans. Antennas Propag., vol. 49, pp. 91-103, 2001.

[18] T. K. Sarkar and J. Koh, "A Pragmatic Approach to Adaptive Antennas," IEEE Antennas Propag. Mag., vol. 42, no. 2, April 2000.

[19] T. Svantesson, "Modeling and estimation of mutual coupling in a uniform linear array of dipoles," Proc. IEEE Int. Conf. Acoustics, Speech, Signal Processing, vol. 5, pp. 2961-2964, March 1999.

\section{Introduction}

Adaptive antenna arrays are useful in communication systems since they can electronically steer the pattern of the antenna to any desired direction. The performance of an adaptive antenna array is strongly affected by the effect of Mutual Coupling (MC) between antenna elements, and ignoring its effect, can seriously degrade the performance of algorithms [1,2]. Research into compensating for the MC has been based mainly on the idea of using open circuit voltages, firstly proposed by Gupta and Ksienski [1]. Many efforts have been made to compensate for the MC in adaptive antenna array $[1,2$, $3,4,5,6,7,8,9,10]$. Unfortunately, the MC matrix tends to change with time due to environmental factors [3], so it is impossible to fully eliminate its effect and predict its variability. Therefore, calibration procedures based on signal processing algorithms are needed to estimate and compensate for the effect of 
MC. The most likely way for calibration is to carry out some measurements. Typically, this is done by using a source that is moved along a known path around the array or by using a set of calibration sources at known positions. However, these procedures have the drawbacks of being time consuming and expensive [11]. Because of such drawbacks, some self calibration algorithms have been proposed to damp the MC effect [12, 13, 14, 15].

In this paper, an adaptive algorithm based on Direct Data Domain Least Squares algorithms $\left(\mathrm{D}^{3} \mathrm{LS}\right)$ including mutual coupling effect is presented. The $\mathrm{D}^{3} \mathrm{LS}$ algorithms $[16,17,18]$ have been proposed to overcome the drawbacks of statistical techniques and utilizing one snapshot makes it possible to carry out an adaptive process in real time. The algorithm is to adaptively recover a desired signal while simultaneously rejecting all other interference. The performance of this algorithm affected by the MC effect, too [5, 6]. Using the $\mathrm{D}^{3} \mathrm{LS}$ algorithm properties, we obtain the coupling coefficients without using any auxiliary sensors.

In Section 2, we investigate the effect of $\mathrm{MC}$ on the performance of a $\mathrm{D}^{3} \mathrm{LS}$ algorithm. In Section 3, we formulate the full effect of MC between whole elements and present a new technique for compensation of the MC effect. In Section 4, numerical simulation illustrates that the proposed algorithm can accurately recover the desired signal in the presence of MC.

\section{Review of $D^{3}$ LS algorithm and the effects of mutual cou- pling}

Consider a linear adaptive array consisting of $N$ equally spaced elements with spacing of $d$. The array receives a signal from a known direction $\theta_{0}$ and $M$ interferers from unknown directions $\theta_{m}, m=1,2, \ldots, M$ as shown in Fig. 1.

Assume that all signal and jammers are narrowband and propagate perpendicularly to the z -direction. Sarkar and Sangruji [16] have been proved that the desired signal can be recovered from adaptive algorithms of direct

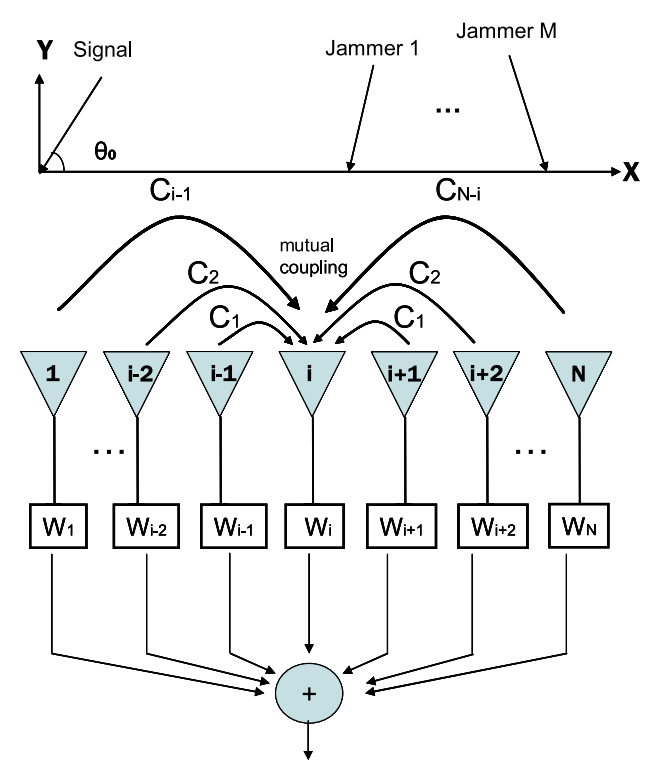

Fig. 1. Linear Uniform Array 
data domain as $M \leq(N-1) / 2$. This technique is based on the fact that in the absence of $\mathrm{MC}$, a far-field source presents a linear phase front at the ports of a linear array. By suppressing time dependence in phasor notation, the output voltage can be expressed as:

$$
\mathbf{x}=S \mathbf{a}\left(\theta_{0}\right)+\left(\sum_{m=1}^{M} J_{m} \mathbf{a}\left(\theta_{m}\right)\right)+\mathbf{n}
$$

Where $\mathbf{x}, \mathbf{a}, \mathbf{n}, S, J_{m}$ denote the received signal vector, the steering vector, additive white gaussian noise vector, the complex amplitude of the desired signal and $m$ th interferers, respectively. They are defined as:

$$
\begin{aligned}
& \mathbf{x}=\left[x_{1}, x_{2}, \ldots, x_{N}\right]^{T}, \mathbf{n}=\left[n_{1}, \ldots, n_{N}\right]^{T} \\
& \mathbf{a}\left(\theta_{i}\right)=\left[1, e^{-j 2 \pi \frac{d}{\lambda} \cos \theta_{i}}, \ldots, e^{-j 2 \pi(N-1) \frac{d}{\lambda} \cos \theta_{i}}\right]^{T} \quad \text { for } \quad i=0,1, \ldots, M
\end{aligned}
$$

The superscript $(\bullet)^{T}$ is transpose operation. Let us define

$$
z=\exp \left(j 2 \pi \frac{d}{\lambda} \cos \theta_{0}\right)
$$

We can see, the term $x_{i}-z^{-1} x_{i+1}$ has no the signal component. Therefore one can form a reduced rank matrix where the weighted sum of all its elements would be zero [16]. The additional equation is introduced through the constraint that the same weights when operating on the signal produced a gain factor $P$, which is the first equation. Then we can write:

$$
\left[\begin{array}{ccc}
1 & \cdots & z^{K-1} \\
x_{1}-z^{-1} x_{2} & \cdots & x_{K}-z^{-1} x_{K+1} \\
\vdots & \vdots & \vdots \\
x_{K-1}-z^{-1} x_{K} & \cdots & x_{N-1}-z^{-1} x_{N}
\end{array}\right]_{K \times K} \times\left[\begin{array}{c}
w_{1} \\
w_{2} \\
\vdots \\
w_{K}
\end{array}\right]=\left[\begin{array}{c}
P \\
0 \\
\vdots \\
0
\end{array}\right]_{K \times 1}
$$

Where $\mathbf{w}=\left[w_{1}, w_{2}, \ldots, w_{K}\right]^{T}$ is the weight vector and $K=(N+1) / 2[18]$. Now, the desired signal can be recovered as [18]:

$$
S=\frac{1}{P} \sum_{k=1}^{K} w_{k} x_{k}
$$

\section{Mutual coupling compensation with the proposed algorithm}

If one assume that $\mathbf{C}$ denotes the mutual coupling matrix of the array, the output will be as:

$$
\mathbf{x}_{\mathbf{c}}=S \mathbf{C a}\left(\theta_{0}\right)+\left(\sum_{m=1}^{M} J_{m} \mathbf{C a}\left(\theta_{m}\right)\right)+\mathbf{n}
$$

Where $\mathbf{x}_{\mathbf{c}}=\left[x_{c 1}, x_{c 2}, \ldots, x_{c N}\right]^{\mathrm{T}}$ denotes, the received voltage vector in the presence of MC. Each sensor is affected by coupling of all sensors around it, as shown in Fig. 1. Ignoring the MC, the term $x_{i}-z^{-1} x_{i+1}$, has no signal 
component, but in the presence of $\mathrm{MC}$, we have

$$
\begin{array}{ll}
x_{c 1}-z^{-1} x_{c 2} & =-S c_{1} z^{-1}+S c_{N-1} z^{N-1}+\text { Interferers } \\
x_{c 2}-z^{-1} x_{c 3} & =-S c_{2} z^{-1}+S c_{N-2} z^{N-1}+\text { Interferers } \\
\ldots & \\
x_{c(N-2)}-z^{-1} x_{c(N-1)} & =-S c_{N-2} z^{-1}+S c_{2} z^{N-1}+\text { Interferers } \\
x_{c(N-1)}-z^{-1} x_{c N} & =-S c_{N-1} z^{-1}+S c_{1} z^{N-1}+\text { Interferers }
\end{array}
$$

Where $c_{i}$ is the coupling between $r$ and $r \pm i$ elements. As we see from (7), it can't be solved to determine the $c_{i}$, because the matrix of equation is singular. It has been shown in [19], that the coupling between neighboring elements of a Uniform Linear Array (ULA) is almost the same, and the magnitude of the coupling parameters decreases very fast along with the increasing sensor spacing. Essentially, mutual coupling coefficient between two far apart elements can be approximated as zero. Thus, it is often sufficient to consider the ULA coupling model that has just finite nonzero coefficients, and a banded symmetric toeplitz matrix can be used as a model for the mutual coupling. We define the MC matrix in order $\mathrm{i}$, if one sensor is affected by coupling of $i$ sensors around it

$$
\mathbf{C}=\left[\begin{array}{cccccccccc}
1 & c_{1} & c_{2} & c_{3} & \ldots & c_{i} & 0 & 0 & 0 \ldots & 0 \\
c_{1} & 1 & c_{1} & c_{2} & \ldots & c_{i-1} & c_{1} & 0 & 0 \ldots & 0 \\
c_{2} & c_{1} & 1 & c_{1} & \ldots & c_{i-2} & c_{i-1} & c_{1} & 0 \ldots & 0 \\
\vdots & \ddots & \ddots & \ddots & \ddots & & \ddots & & \vdots \\
0 & \ldots & 0 & 0 & c_{i} & c_{i-1} & \ldots & c_{1} & 1 & c_{1} \\
0 & \ldots & 0 & c_{i} & c_{i-1} & c_{i-2} & \ldots & c_{2} & c_{1} & 1
\end{array}\right]_{N \times N}
$$

Here, we suppose, $c_{i}=0, i=K, K+1, \ldots, N$. Then (7) is simplified and we can solve it as $i=1,2, \ldots, K-1$ :

$$
\begin{aligned}
& x_{c 1}-z^{-1} x_{c 2} \quad=-S c_{1} z^{-1} \quad+\text { Noise }+ \text { Interferers } \\
& x_{c 2}-z^{-1} x_{c 3} \quad=-S c_{2} z^{-1} \quad+\text { Noise }+ \text { Interferers } \\
& \text {... } \\
& x_{K-1}-z^{-1} x_{c K} \quad=-S c_{K-1} z^{-1} \quad+\text { Noise }+ \text { Interferers } \\
& x_{c K}-z^{-1} x_{c(K+1)}=S c_{K-1} z^{N-1}+\text { Noise }+ \text { Interferers } \\
& x_{c(N-2)}-z^{-1} x_{c(N-1)}=S c_{2} z^{N-1} \quad+\text { Noise }+ \text { Interferers } \\
& x_{c(N-1)}-z^{-1} x_{c N}=S c_{1} z^{N-1} \quad+\text { Noise }+ \text { Interferers }
\end{aligned}
$$

When no interferers, from (9), we can estimate the coupling coefficients:

$$
\begin{aligned}
\frac{c_{2}}{c_{1}}=\frac{\left(x_{c 2}-z^{-1} x_{c 3}\right)}{\left(x_{c 1}-z^{-1} x_{c 2}\right)}, \frac{c_{3}}{c_{1}} & =\frac{\left(x_{c 3}-z^{-1} x_{c 4}\right)}{\left(x_{c 1}-z^{-1} x_{c 2}\right)}, \ldots, \frac{c_{K}}{c_{1}}=\frac{\left(x_{c K}-z^{-1} x_{c(K+1)}\right)}{\left(x_{c 1}-z^{-1} x_{c 2}\right)} \\
\frac{x_{c 1}}{x_{c 2}-z x_{c 1}} & =\frac{\left(1+c_{1} z+c_{2} z^{2}+\ldots+c_{K} z^{K}\right) S}{S c_{1}} \\
& =\left(\frac{1}{c_{1}}+z+\frac{c_{2}}{c_{1}} z^{2}+\ldots+\frac{c_{K}}{c_{1}} z^{K}\right)
\end{aligned}
$$


From (10) and (11), we derived a new formulation for estimate of the $c_{1}$ as:

$$
\frac{1}{c_{1}}=\frac{x_{c 1}}{x_{c 2}-z x_{c 1}}-z-\frac{\left(x_{c 2}-z^{-1} x_{c 3}\right)}{\left(x_{c 1}-z^{-1} x_{c 2}\right)} z^{2}-\ldots-\frac{\left(x_{c K}-z^{-1} x_{c(K+1)}\right)}{\left(x_{c 1}-z^{-1} x_{c 2}\right)} z^{K}
$$

With use of Eqs. (10), we can estimate $c_{2}, c_{3}, \ldots, c_{K}$, and the amplitude of the signal determined as the following:

$$
\tilde{S}=\frac{1}{P}\left(\mathbf{w}_{\mathbf{c}}^{T}\left[\tilde{\mathbf{C}}^{-1} \mathbf{x}_{\mathbf{c}}\right]_{K}\right)
$$

Where $\mathbf{w}_{\mathbf{c}}=\left[w_{c 1}, w_{c 2}, \ldots, w_{c K}\right]^{T}$ is the weight vector in the presence of MC. $\tilde{S}, \tilde{\mathbf{C}}$ are the estimation of $S, \mathbf{C}$, and $[\bullet]_{K}$ denote, the $K$ rows from vector.

\section{Numerical example}

In this section, the method of moments (MoM) is used to accurately model the interactions between antenna elements and then capability of the MC compensation for the proposed algorithm for different orders will be test. A linear array consisting of fifteen z-direction parallel identical dipoles, equally spaced with spacing of $0.5 \lambda$, illuminated by one desired signal and three jammers is considered. Where $\lambda$ is the wavelength. Each dipole is $0.5 \lambda$ long and radius is $\lambda / 200$. All the elements are loaded with a terminal load $\mathrm{Z}_{\mathrm{L}}=$ $50 \Omega$. The desired signal varies from $1 \mathrm{~V} / \mathrm{m}$ to $10 \mathrm{~V} / \mathrm{m}$ and there are three jammers with $100 \mathrm{~V} / \mathrm{m}$ constant intensities and received from $15^{\circ}, 30^{\circ}$ and $60^{\circ}$. The signal-to-noise ratio (SNR) was set at $20 \mathrm{~dB}$. Fig. 2 shows the result of using the new proposed algorithm with different orders estimation in the presence of MC.

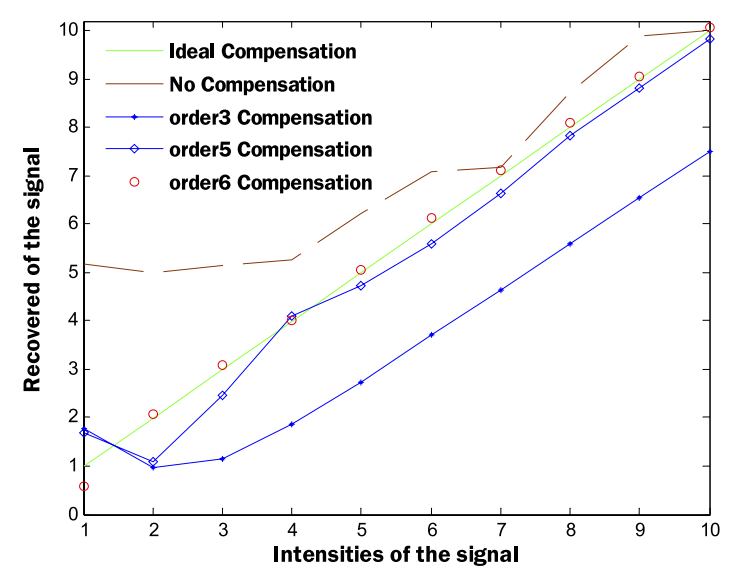

Fig. 2. Recovered of the desired signal using the proposed algorithm with different orders mutual coupling compensation

As we see in Fig. 2, the $\mathrm{D}^{3} \mathrm{LS}$ algorithm couldn't recover the signal accurately in the presence of $\mathrm{MC}$, but the expected linear relationship for the proposed algorithm is clearly seen, implying that the jammers have been nulled and the signal recovered correctly. 


\section{Conclusion}

In this paper, we have studied the problem of $\mathrm{D}^{3} \mathrm{LS}$ algorithms in the presence of mutual coupling. With the use of $\mathrm{D}^{3} \mathrm{LS}$ algorithms properties, we estimate the mutual coupling coefficients. Without the use of moment methods and calculation of impedance matrix, we can estimate the MC automatically and eliminate it and recovered the desired signal accurately. 